\title{
CONTENT BASED IMAGE RETRIEVAL : A REVIEW
}

\author{
Shereena V.B. ${ }^{1}$ and Julie M. David ${ }^{2}$ \\ ${ }^{1,2}$ Asst.Professor, Dept of Computer Applications, \\ MES College, Marampally, Aluva, Cochin, India \\ ${ }^{1}$ shereenavb@gmail.com \\ 2julieeldhosem@yahoo.com
}

\begin{abstract}
In a content-based image retrieval system (CBIR), the main issue is to extract the image features that effectively represent the image contents in a database. Such an extraction requires a detailed evaluation of retrieval performance of image features. This paper presents a review of fundamental aspects of content based image retrieval including feature extraction of color and texture features. Commonly used color features including color moments, color histogram and color correlogram and Gabor texture are compared. The paper reviews the increase in efficiency of image retrieval when the color and texture features are combined. The similarity measures based on which matches are made and images are retrieved are also discussed. The paper discusses effective indexing and fast searching of images based on visual features.
\end{abstract}

\section{KEYWORDS}

CBIR, Color moments, Color histogram, Color correlogram, Gabor filter, Precision, Recall.

\section{INTRODUCTION}

Image Processing involves changing the nature of an image in order to improve its pictorial information for human interpretation and render it more suitable for autonomous machine perception [1].The advantage of image processing machines over humans is that they cover almost the entire electromagnetic spectrum, ranging from gamma to radio waves where as human eye is limited to the visual band of the electromagnetic spectrum. They can operate on images generated by sources like ultrasound, electron microscopy, and computer-generated images. Thus image processing has an enormous range of applications and almost every area of science and technology such as medicine, space program, agriculture, industry and law enforcement make use of these methods. One of the key issues with any kind of image processing is image retrieval which is the need to extract useful information from the raw data such as recognizing the presence of particular color or textures before any kind of reasoning about the image's contents is possible.

Early work on image retrieval can be traced back to the late 1970s. In 1979, a conference on Database Techniques for Pictorial Applications was held in Florence[2]. Early techniques were not generally based on visual features but on the textual annotation of images, where traditional database techniques are used to manage images. Many difficulties were faced by text based retrieval, since volume of digital images available to users increased dramatically. The efficient management of the rapidly expanding visual information became an urgent problem. This need

David C. Wyld et al. (Eds) : COSIT, DMIN, SIGL, CYBI, NMCT, AIAPP - 2014

pp. 65-77, 2014. (C) CS \& IT-CSCP 2014

DOI : $10.5121 /$ csit.2014.4906 
formed the driving force behind the emergence of content-based image retrieval techniques (CBIR).

CBIR is a technique which uses visual contents to search images from an image database. In CBIR, visual features such as colour and texture are extracted to characterise images. CBIR draws many of its methods from the field of image processing and computer vision, and is regarded as a subset of that field. In CBIR, visual contents are extracted and described by multidimensional feature vectors. To retrieve images, users provide the retrieval system with example images. The system changes them into internal representation of feature vectors. The similarities or differences between feature vectors of the query examples and those of the images in the database are calculated and retrieval performed with an indexing scheme .The indexing scheme is an efficient way to search for image database. Recent retrieval systems have incorporated user's relevance feedback to modify the retrieval process.

The tasks performed by CBIR can be classified into pre-processing and feature extraction stages. In Pre-processing stage, removal of noise and enhancement of some object features which are relevant to understanding the image is performed. Image segmentation is also performed to separate objects from the image background. In Feature Extraction stage, features such as shape, colour, texture etc. are used to describe the content of the image. This feature is generated to accurately represent the image in the database. The colour aspect can be achieved by the techniques like moments, histograms and correlograms. The texture aspect can be achieved by using transforms or vector quantization. Similarity Measurement is also done in this stage. ie. the distance between query image and different images in the database is calculated and the one with the shorter distance is selected [3]. Similarity measurement can be formulated as follows.

Let $\{F(x, y): x=1,2, \ldots X, y=1,2, \ldots Y\}$ be a $2 D$ image pixel array.

For colorimages , $\mathrm{F}(\mathrm{x}, \mathrm{y})$ denotes the color value at pixel $(\mathrm{x}, \mathrm{y})$ ie, $\{\mathrm{F}(\mathrm{x}, \mathrm{y})=\mathrm{FR}(\mathrm{x}, \mathrm{y}), \mathrm{FG}(\mathrm{x}, \mathrm{y}), \mathrm{FB}(\mathrm{x}, \mathrm{y})\}$

For black and white images $\mathrm{F}(\mathrm{x}, \mathrm{y})$ denotes the gray scale intensity at $(\mathrm{x}, \mathrm{y})$.

The problem of image retrieval can be quoted mathematically as follows:

For a query image $\mathrm{Q}$, we find an image $\mathrm{T}$ from the image database such that the distance between corresponding feature vectors is less than the specified threshold $t$.

$$
\text { ie,D(Feature(Q),Feature }(\mathrm{T}))<=\mathrm{t}
$$

There is a lot of research being done in the field of CBIR in order to generate better methodologies for feature extraction. In this paper, a study of different color and texture descriptors for content-based image retrieval is carried out to find out whether a combination of different features gives better results.

The rest of this paper is organized as follows. In Section 2, we discuss previous work in CBIR. In Section 3, we explain feature extraction and representation methods. Section 4 explains combination of features, Section 5 explains Performance evaluation and indexing schemes and finally, conclusions are given in Section 6. 


\section{LITERATURE REVIEW}

Researchers have proposed different methods to improve the system of content based image retrieval. Ryszard S. Chora's[3]stated in his paper that the similarity of the feature vectors of the query and database images is measured to retrieve the image. M. Stricker, and M. Orengo, have shown that[4] the first order (mean), the second (variance) and the third order (skewness) color moments have been proved to be efficient and effective in representing color distributions of images. In his paper J. Huang, et al., [5] proposed the color correlogram to characterize not only the color distributions of pixels, but also the spatial correlation of pairs of colors. Deepak $\mathrm{S}$. Shete1, Dr. M.S. Chavan[6] proposed that the ability to match on texture similarity can often be useful in distinguishing between areas of images with similar color (such as sky and sea, or leaves and grass). Fazal Malik, Baharum Baharudin[7] proposed a CBIR method which is based on the performance analysis of various distance metrics using the quantized histogram statistical texture features. The similarity measurement is performed by using seven distance metrics. The experimental results are analysed on the basis of seven distance metrics separately using different quantized histogram bins such that the Euclidean distance has better efficiency in computation and effective retrieval. This distance metric is most commonly used for similarity measurement in image retrieval because of its efficiency and effectiveness.

In the paper of Manimala Singhaand K.Hemachandran [8], they presented a novel approach for Content Based Image Retrieval by combining the color and texture features called Wavelet-Based Color Histogram Image Retrieval (WBCHIR). Similarity between the images is ascertained by means of a distance function. The experimental result shows that the proposed method outperforms the other retrieval methods in terms of Average Precision. Md. Iqbal Hasan Sarker and Md. ShahedIqbal [9] proposed that using only a single feature for image retrieval may be inefficient. They used color moments and texture features and their experiment results demonstrated that the proposed method has higher retrieval accuracy than the other methods based on single feature extraction. N.R. Janani and Sebhakumar P. suggests [10] a content-based image retrieval method which combines color and texture features in order to improve the discriminating power of color indexing techniques and also a minimal amount of spatial information is encoded in the color index. The motivation behind this paper is a study on the works done by early researchers in the field of content based image retrieval based on color and texture features.

\section{FEATURE EXTRACTION AND REPRESENTATION}

Features are properties of images such as colour, texture, shape, edge information extracted with image processing algorithms. A single feature does not give accurate results, but a combination of features is minimally needed to get accurate retrieval results.

\subsection{Color}

The most widely used visual feature in image retrieval is color feature. Color feature is relatively robust to background complications. Each pixel can be represented as a point in 3D color space. Commonly used color space include RGB, CIELab where "L" value for each scale indicates the level of light or dark, "a" value redness or greenness, and "b" value yellowness or blueness, HSV (Hue, Saturation, Value).

In the RGB color space, a color is represented by a triplet $(R, G, B)$, where $R$ gives the intensity of the red component, $\mathrm{G}$ gives the intensity of the green component and B gives the intensity of the blue component. The CIE Lab spaces are device independent and considered to be perceptually 
uniform. They consist of a luminance or lightness component (L) and two chromatic components $\mathrm{a}$ and $\mathrm{b}$ or $\mathrm{u}$ and $\mathrm{v}$. HSV (or HSL, or HSB) space is widely used in computer graphics and is a more intuitive way of describing color. The three color components are hue, saturation(lightness) and value (brightness). HSV colour model describes colours in terms of their shades and brightness (Luminance). This model offers a more intuitive representation of relationship between colours. Basically a colour model is the specification of coordinate system and a subspace within that, where each colour is represented in single point. Hue represents the dominant wavelength in light. It is the term for the pure spectrum colours. Hue is expressed from $0^{\circ}$ to $360^{\circ}$. It represents hues of red (starts at $0^{\circ}$ ),yellow (starts at $60^{\circ}$ ), green (starts at $120^{\circ}$ ), cyan (starts at $180^{\circ}$ ), blue (starts at $240^{\circ}$ ) and magenta (starts at $300^{\circ}$ ). Eventually all hues can be mixed from three basic hues known as primaries. Saturation represents the dominance of hue in colour. It can also be thought as the intensity of the colour. It is defined as the degree of purity of colour. A highly saturated colour is vivid, whereas a low saturated colour is muted. When there is no saturation in the image, then the image is said to be a grey image. Value describes the brightness or intensity of the colour. It can also be defined as a relative lightness or darkness of colour [11]. The HSV values of a pixel can be transformed from its RGB representation according to the following formula:

$$
\begin{aligned}
& H=\cos ^{-1} \frac{1}{2} \frac{(R-G)+(R-B)]}{\sqrt{\left[(R-G)^{2}+(R-B)(G-B)\right]}} \\
& S=1-\frac{3[\min (R, G, B)]}{R+G+B} \quad V=\frac{R+G+B}{3}
\end{aligned}
$$

Once the colour space is specified, colour feature is extracted from images or regions. A number of important colour features have been proposed in the literatures, including color moments (CM),color histogram, color correlogram etc. The Color moment can be used as remedies of user's queries which are semantic in nature. Color histogram is a popular color feature that has been widely used in many image retrieval systems. Color histogram is robust with respect to viewpoint axis and size, occlusion, slow change in angle of vision and rotation. The color correlogram was proposed to characterize not only the color distributions of pixels, but also the spatial correlation of pairs of colors. Compared to the color histogram the color correlogram provides the best retrieval results, but is also the most computational expensive due to its high dimensionality.

\subsubsection{Color moments}

To differentiate objects based on color, Color moments have been successfully used in many retrieval systems, especially when the image contains just the object. The basis of color moments is that the distribution of color in an image can be considered as a probability distribution which can be characterized by various moments. ie. If the color in an image follows a certain probability distribution, the image can be identified by that distribution using moments. The first order (mean), the second order (variance) and the third order (skewness) color moments have been proved to be efficient and effective in representing color distributions of images[4].

$$
\begin{gathered}
\mu_{i}=\frac{1}{N} \sum_{j=1}^{n} P_{i j} \\
\sigma_{i}=\left(\frac{1}{N} \sum_{j=1}^{n}\left(\left(P_{i j}-\mu_{i}\right)^{2}\right)^{\frac{1}{2}}\right.
\end{gathered}
$$




$$
S_{i}=\left(\frac{1}{N} \sum_{j=1}^{n}\left(\left(P_{i j}-\mu_{i}\right)^{3}\right)^{\frac{1}{3}}\right.
$$

Where $\mathrm{P}_{\mathrm{ij}}$ is the value of the $\mathrm{i}$ - the color channel of image pixel $\mathrm{j}$ and $\mathrm{N}$ is the number of pixels in the image.

A color can be defined by 3 or more values. Here we can use any of the color coding schemes, say HSV. A moment can be calculated for each of these channels. Thus we get nine numbersthree moments for each color channel as color features for each of the image. Thus color moments are a very compact representation compared to other color features. Due to this compactness, it may also lower the discrimination power.

Similarity between two image distributions is defined as the sum of weighted differences between the moments of two distributions.

ie.

$$
d_{m o m}(H, I)=\sum_{i=1}^{r} w_{i 1}\left|E_{i}^{1}-E_{i}^{2}\right|+w_{i 2}\left|\sigma_{i}^{1}-\sigma_{i}^{2}\right|+w_{i 3}\left|S_{i}^{1}-S_{i}^{2}\right|
$$

where $(\mathrm{H}, \mathrm{I})$ are the two image distribution components, $\mathrm{i}$ is the current channel index $(1=\mathrm{H}, 2=\mathrm{S}, 3=\mathrm{V}), \mathrm{r}$ is the number of channels ,here $3, E_{i}^{1}, E_{i}^{2}$ are the first order moments of two image distributions, $\sigma_{i}^{1}, \sigma_{i}^{2}$ are the second order moments of two image distributions, $S_{i}^{1}, S_{i}^{2}$ are the third order moments of the two image distributions and $w_{i}$ are the weights for each moment. Pairs of images are ranked based on $d_{\text {mom }}$ values. The images with lower $d_{\text {mom }}$ values are ranked high and are more similar compared to those with higher $d_{m o m}$ values.

The methodology used to calculate moments is as follows. We first scale all images to the same size for efficiency. Color moments are based on probability distributions. So image size should not change the result of comparison. We calculate the three color moments using the formula defined above for the Query Image. We then repeat the calculations for our database images. Calculate $d_{m o m}$ value after giving appropriate weights and rank the images in the increasing order of this value. The images with the lowest $d_{\text {mom }}$ values are selected as the result images. In this way, we can use color moments as a technique to compare images based on color. Color moments can be used as the first pass to narrow down the search space before other sophisticated color features are used for retrieval.

\subsubsection{Color Histogram}

Color Histogram represents the distribution of intensity of the colorin the image. Color histograms are a set of bins where each bin denotes the probability of pixels in the image being of a particular color. It serves as an effective representation of the color content of an image if the color pattern is unique compared with the rest of the data set. In addition, it is robust to translation and rotation about the view axis and changes only slowly with the scale, occlusion and viewing angle [3].

A color histogram $\mathrm{H}$ for a given image is defined as a vector

$\mathrm{H}=\{\mathrm{H}[1], \mathrm{H}[2], \ldots \mathrm{H}[\mathrm{i}], \ldots, \mathrm{H}[\mathrm{N}]\}$ 
where $\mathrm{i}$ represent a color in the color histogram, $\mathrm{H}[\mathrm{i}]$ is the number of pixels in color $\mathrm{i}$ in that image, and $\mathrm{N}$ is the number of bins in thecolor histogram, i.e., the number of colors in the adopted color model.

In order to compare images of different sizes, color histograms should be normalized. The normalized color histogram $\mathrm{H}^{\prime}$ is defined as

$$
H^{\prime}=\left\{H^{\prime}[0], H^{\prime}[1], \ldots H^{\prime}[i], \ldots H^{\prime}[N]\right\}
$$

where $H^{\prime}[i]=\frac{H[i]}{X Y}, X Y$ is the total number of pixels in an image.

An ideal color space quantization presumes that distinct colors should not be located in the same sub-cube and similar colors should be assigned to the same sub-cube. A color histogram with few colors will decrease the possibility that similar colors are assigned to different bins, but it increases the possibility that distinct colors are assigned to the same bins, and that the information content of the images will decrease by a greater degree. color histograms with a large number of bins will contain more information about the content of images, thus decreasing the possibility of distinct colors will be assigned to the same bins.

Minkowski-form distance metrics [12] compare only the same bins between color histograms and are defined as:

$$
d(Q, I)=\sum_{i=1}^{N}\left|H_{Q}[i]-H_{I}[i]\right|^{r}
$$

Where $\mathrm{Q}$ and $\mathrm{I}$ are two images, $\mathrm{N}$ is the number of bins in the color histogram (for each image we reduce the colors to $\mathrm{N}$, in the RGB color space, so each color histogram has $\mathrm{N}$ bins), $H_{Q}[i]$ is the value of bin i in color histogram $H_{Q}$, which represents the image $\mathrm{Q}$, and $H_{I}[i]$ is the value of bin $\mathrm{i}$ in color histogram $H_{I}$ which represents the image I.

When $r=1$, the Minkowski-form distance metric becomes $\mathrm{L}_{1}$. When $\mathrm{r}=2$, the Minkoski-form distance metric becomes the Euclidean distance. This Euclidean distance can be treated as the spatial distance in a multi-dimensional space. In this paper, we will use the square root of Euclidean distance to calculate the distance between two color histograms, which is defined as:

$$
d(Q, I)=\sqrt{ } \sum_{i=1}^{N}\left|H_{Q}[i]-H_{I}[i]\right|^{2}
$$

The image retrieval using histogram consists of the following stages. First of all Query image is given from the user. Then the histogram of the color image is calculated. Each image added to the database is analysed and a colour histogram is computed which shows the proportion of pixels of each colour within the image. Then this colour histogram for each image is stored in the database. Finally Euclidean Distance from query image to database images is calculated and sorted the distance in ascending order and the top images are displayed on the screen. Thus we can use color histograms to retrieve matching images from the database. It performs well compared to other descriptors when images have mostly uniform color distribution but it has the disadvantages of lack of spatial information and therefore tends to give poor results. If two images have exactly the same color proportion but the colors are scattered differently, then we can't retrieve correct images using color histogram. 


\subsubsection{Color correlogram}

A color correlogram is a table indexed by color pairs, where the k-th entry for $(i, j)$ specifies the probability off inding a pixel of color $\mathrm{j}$ at a distance $\mathrm{k}$ from a pixel of color $\mathrm{i}$ in the image[5]. Let I represent the entire set of image pixels and $\mathrm{I}_{\mathrm{c}(\mathrm{i})}$ represent the set of pixels whose colors are c(i).Then, the color correlogram is defined as:

$$
\gamma_{(i, j)}(k)=\operatorname{Pr}_{p 1 \in c(I), p 2 \in I}\left[p 2 \in I_{c(j)}|p 1-p 2|=k\right]
$$

Where $i, j \in\{1,2, \ldots, N\}, k \in\{1,2, \ldots, d\}$, and $|\mathrm{p} 1-\mathrm{p} 2|$ is the distance between pixels $p 1$ and p2.

If we consider all the possible combinations of color pairs the size of the color correlogram will be very large $(\mathrm{O}(\mathrm{N} 2 \mathrm{~d}))$, therefore a simplified version of the feature called the color auto correlogram is often used instead. The color auto correlogram only captures the spatial correlation between identical colors and thus reduces the dimension to $\mathrm{O}(\mathrm{Nd})[5]$.

L1 and L2 distance metrics in Minkowski-form distance metrics [12] are used to compare color features of two images. For correlograms, L1 is used in most cases because it is simple and robust. The distance between two images I and I' is calculated as follows:

$$
\begin{gathered}
\left|I-I^{\prime}\right|_{h, L 1}=\sum_{i \in[m]}\left|h_{c_{i}}(I)-h_{c_{i}}\left(I^{\prime}\right)\right| \\
\left|I-I^{\prime}\right|_{\gamma, L 1}=\sum_{i, j \in[m], k \in[d]}\left|\gamma_{c_{i}, c_{j}(K)},(I)-\gamma_{c_{i}, c_{j}(K)},\left(I^{\prime}\right)\right|
\end{gathered}
$$

The image retrieval problem in color correlogram is as follows. A Query image is given from the user. Then the correlogram of the color image is calculated. Color correlograms of the database images are also calculated. Then the distance from query image to database images is calculated using L1 metric and sorted the distance in ascending order and the top images are displayed on the screen. Thus we can use color correlograms to retrieve matching images from the database.

\subsection{Texture}

Texture is another property of image which is used in pattern recognition and computer vision. Texture [13] is defined as structure of surfaces formed by repeating a particular element or several elements in different relative spatial positions. The repetition involves local variations of scale, orientation, or other geometric and optical features of the elements. The ability to match on texture similarity can often be useful in distinguishing between areas of images with similar color (such as sky and sea, or leaves and grass)[6].Thus texture analysis plays an important role in comparison of images supplementing the color feature. Texture representation methods can be classified into Structural and Statistical categories. Structural methods are applied to textures that are very regular. Statistical methods, includes characterizing texture by the statistical distribution of the image intensity.

Many Statistical techniques has been used for measuring texture similarity in which the best established rely on comparing values of second order statistics calculated from query and stored images [11]. These techniques calculate the relative brightness of selected pairs of pixels from each image. From these it is possible to calculate measures of image texture such as the degree of contrast, coarseness, directionality and regularity, or periodicity, directionality and randomness. Alternative methods of texture analysis for retrieval include the use of Gabor filters and Wavelets. 
Texture queries can be formulated in a similar manner to colour queries, by selecting examples of desired textures from a palette, or by supplying an example query image.

\subsubsection{Gabor filter}

The Gabor filter is a statistical method that has been widely used to extract texture features [14]. This is the most frequently used method in image retrieval by texture. There have been many approaches proposed to characterize textures of images based on Gabor filters. In most of the CBIR systems based in Gabor wavelet, the mean and standard deviation of the distribution of the wavelet transform coefficients are used to construct the feature vector [15].

The basic idea of using Gabor filters to extract texture features is as follows.

A two dimensional Gabor function $g(x, y)$ is defined as:

$$
g(x, y)=\frac{1}{2 \pi \sigma_{x} \sigma_{y}} \exp \left[-\left(\frac{1}{2}\right)\left(\left(\frac{x^{2}}{\sigma_{x}^{2}}\right)+\left(\frac{y^{2}}{\sigma_{y}^{2}}\right)+2 \pi j w_{x}\right)\right]
$$

Where $\sigma_{x}$ and $\sigma_{y}$ are the standard deviations of the Gaussian envelopes along the $\mathrm{x}$ and $\mathrm{y}$ direction.

Given and image $\mathrm{I}(\mathrm{x}, \mathrm{y})$ it's Gabor transform is defined as

$$
w_{m n}(x, y)=\int I(x, y) g_{m n}^{*}\left(x-x_{1}, y-y_{1}\right) d x_{1} d y_{1}
$$

Where $*$ indicates the complex conjugate. Then the mean $\mu_{m n}$ and the standard deviation $\sigma_{m n}$ of the magnitude of $w_{m n}(x, y)$

i.e. $\mathrm{f}=\left[\mu_{00,}, \sigma_{00, \ldots . . .} \mu_{m n}, \sigma_{m n}, \mu_{s-1 k-1}, \sigma_{s-1 k-1}\right]$ can be used to represent the feature of a homogeneous texture region.

The texture similarity measurement of a query image $\mathrm{Q}$ and a target image $\mathrm{T}$ in the database is defined by

$d(Q, T)=\sum_{m} \sum_{n} d_{m n}(Q, T)$

Where $d_{m n}=\frac{\left|\left(\mu_{m n}^{Q}-\mu_{m n}^{T}\right)\right|}{\left|\mu_{m n}^{Q}\right|+\left|\mu_{m n}^{T}\right|}+\frac{\left|\left(\sigma_{m n}^{Q}-\sigma_{m n}^{T}\right)\right|}{\left|\sigma_{m n}^{Q}\right|+\left|\sigma_{m n}^{T}\right|}$

If $\mathrm{f}_{\mathrm{g}}^{\mathrm{Q}}=\left[\mu_{00}, \sigma_{00}, \mu_{01}, \sigma_{01} \ldots . ., \mu_{35}, \sigma_{35}\right]$ denote texture feature vector of query image $\operatorname{andf}_{\mathrm{g}}{ }^{\mathrm{T}}=\left[\mu_{00}, \sigma_{00}, \mu_{01}, \sigma_{01} \ldots \ldots, \mu_{35}, \sigma_{35}\right]$ denote texture feature vector of database image, then distance between them is given by:

$$
d_{2}=\sum_{i=1}^{d} \frac{\left|f_{g}^{Q}-f_{g}^{T}\right|}{\left|f_{g}^{Q}\right|-\left|f_{g}^{T}\right|}
$$

The Canberra distance measure is used for similarity expression. In the case of low level texture feature, we apply Gabor filters on the query image and we obtain an array of magnitudes. The 
mean $\mu_{m n}$ and standard deviation $\sigma_{m n}$ of the magnitudes are used to create a texture feature vector $f_{g}$. Similarly the Gabor filters of database images are also calculated and Canberra distance measure is used for computing the distance between query and database images and the results of a query are displayed in decreasing similarity order. In this way Gabor filter can be used to match images from the database using texture property of the image.

\subsubsection{Haar wavelet Transforms}

Wavelet transforms provide a multi-resolution approach to texture analysis and classification. The wavelet transform represents a function as a superposition of a family of basic functions called wavelets. The wavelet transform computation of a two-dimensional image is also a multiresolution approach, which applies recursive filtering and sub-sampling. At each level, the image is decomposed into four frequency sub-bands, LL, LH, HL, and $\mathrm{HH}$ where $\mathrm{L}$ denotes low frequency and $\mathrm{H}$ denotes high frequency.

If a data set $X_{0}, X_{1, \ldots} X_{n-1} \ldots$ Contains $N$ elements [9], there will be $N / 2$ averages and $N / 2$ wavelet coefficient values. The averages are stored in the first half of the $\mathrm{N}$ element array, and the coefficients are stored in the second half of the $\mathrm{N}$ element array. The averages become the input for the next step in the wavelet calculation. The Haar equations to calculate an average and a wavelet coefficient from an odd and even element in the data set are

$$
\begin{aligned}
& a_{i}=\frac{\left(X_{i}+X_{i+1}\right)}{2} \\
& c_{i}=\frac{\left(X_{i}-X_{i+1}\right)}{2}
\end{aligned}
$$

For a 1D Haar transform of an array of $\mathrm{N}$ elements, find the average of each pair of elements, find the difference between each pair of elements and divide it by 2, fill the first half of the array with averages, fill the second half of the array with coefficients and Repeat the process on an average part of the array until a single average and a single coefficient are calculated. For a 2D Haar transform, Compute 1D Haar wavelet decomposition of each row of the original pixel values and then compute1D Haar wavelet decomposition of each column of the row-transformed pixels. Red, green and blue values are extracted from the images. Then we apply the 2D Haar transform to each color matrix.

We apply Haar wavelet decomposition of an image in the RGB color space. We continue decomposition up to level 4 , and with F-norm theory we decrease the dimensions of image features and perform highly efficient image matching. If $A$ is a square matrix and $A_{i}$ is its $i^{\text {th }}$ order sub-matrix where

$$
A=\left[\begin{array}{l}
a_{11}, \ldots a_{1 n} \\
a_{n 1}, \ldots a_{n n}
\end{array}\right] A_{i}=\left[\begin{array}{l}
a_{11}, \ldots a_{1 i} \\
a_{i 1}, \ldots a_{i i}
\end{array}\right] \quad i=1 \sim n
$$

F-norm of $\mathrm{A}_{i}$ is

$\left\|A_{i}\right\|_{F}-\| A_{i-1}||_{F}$ and ||$A_{i} \|_{F}=0$, we can define feature vector of $\mathrm{A}$ as

$$
\left\{V_{A F}=\left\{\Delta A_{1}, \Delta A_{2}, \ldots . \Delta A_{n}\right\}\right.
$$

The similarity between two images is computed by calculating the distance between feature representation of the query image and feature representation of the image in the dataset. We use Canberra distance for distance calculation of the feature vectors. 


$$
d(q, d)=\sum_{i=1}^{n} \frac{\left|q_{i}-d_{i}\right|}{\left|q_{i}\right|-\left|d_{i}\right|}, \text { where }
$$

$\mathrm{q}=\left(\mathrm{q}_{1}, \mathrm{q}_{2}, \ldots \mathrm{q}_{\mathrm{n}}\right)$ is the feature vector of the query image,

$d=\left(d_{1}, d_{2, \ldots} d_{n}\right)$ is the feature vector of the image in the database,

$\mathrm{n}=$ number of elements of the feature vector.

A feature vector is extracted from each image in the database and the set of all feature vectors is organized as a database index. When similar images are searched with a query image, a feature vector is extracted from the query image and is matched against the feature vectors in the index. If the distance between feature representation of the query image and feature representation of the database image is small, then it is considered similar. Thus we can use Haar wavelet for matching images from the database.

\section{COMbining THE FEATURES}

The image retrieval using only single feature such as color moment or color histogram may be inefficient. It may either retrieve images not similar to query image or may fail to retrieve images similar to query image. To produce efficient results, we use combination of color and texture features. The similarity between query and target image is measured from two types of characteristic features which includes color and texture features. Two types of characteristics of images represent different aspects of property. While calculating similarity measure, appropriate weights are considered to combine the features [9]. The distance between the query image and the image in the database is calculated as follows:

$$
\mathrm{d}=\mathrm{w} 1 * \mathrm{~d} 1+\mathrm{w} 2 * \mathrm{~d} 2 .
$$

Here, w1 is the weight of the color features, w 2 is the weight of the texture features and $\mathrm{d} 1$ and $\mathrm{d} 2$ are the distances calculated using color features and texture features respectively. The distance $d$ is calculated for each query image with all images in the database. The image that has a lower distance value is considered the similar image and the results are ranked in the ascending order of d. From the studies, [16] It is seen that the value of the average precisions based on single features i.e. only Gabor texture features or only Color moments are less than the average precisions of combined features of color moments and Gabor texture features. This shows that there is considerable increase in retrieval efficiency when both color and texture features are combined for CBIR. Also it is found that [8] the texture and color features are extracted through wavelet transformation and color histogram and the combination of these features is a faster retrieval method which is robust to scaling and translation of objects in an image.

\section{PERformance EVAluation AND INDEXING SCHEMES}

The performance of retrieval of the system can be measured in terms of its recall and precision. Recall measures the ability of the system to retrieve all the models that are relevant, while precision measures the ability of the system to retrieve only the models that are relevant [8].

$$
\begin{gathered}
\text { Precision }=\frac{\text { Number of relevant images retrieved }}{\text { Total Number of images retrieved }} \\
\text { Recall }=\frac{\text { Number of relevant images retrieved }}{\text { Total no of relevant images }}
\end{gathered}
$$


where A represent the number of relevant images that are retrieved, B, the number of irrelevant items and the $\mathrm{C}$, number of relevant items those were not retrieved. The number of relevant items retrieved is the number of the returned images that are similar to the query image in this case. The total number of items retrieved is the number of images that are returned by the search engine. In precision and recall, crossover is the point on the graph where the both precision and recall curves meet. The higher the number of crossover points better will be the performance of the system.

The average precision for the images that belongs to the qth category $\left(\mathrm{A}_{\mathrm{q}}\right)$ has been computed by

$$
P^{\prime}=\sum_{k \in A_{q}} \frac{P\left(i_{k}\right)}{\left|A_{q}\right|} \text { Where } \mathrm{q}=1,2 \ldots \ldots 10 .
$$

Finally, the average precision is given by:

$$
P^{\prime}=\sum_{q=1}^{10}\left(P_{q}^{\prime} / 10\right)
$$

Another important issue in content-based image retrieval is effective indexing and fast searching of images based on visual features. The feature vectors of images tend to have high dimensionality and are not well suited to traditional indexing structures. Sodimension reduction is usually used before setting up an efficient indexing scheme. One of the techniques commonly used for dimension reduction is principal component analysis (PCA).It is a general and very recognizable method [17] and an optimal technique that linearly maps the input data to a coordinate space such that the axes are aligned to reflect the maximum variations in the data. The QBIC system uses PCA to reduce a 20-dimensional shape feature vector to two or three dimensions [18].

After dimension reduction, the multi-dimensional data are indexed. A number of approaches have been proposed for this purpose, including R-tree [19], linear quad-trees [20]. Most of these multidimensional indexing methods have reasonable performance for a small number of dimensions (up to 20), but explore exponentially with the increasing of the dimension ality and eventually reduce to sequential searching. Furthermore, these indexing schemes assume that the underlying feature comparison is based on the Euclidean distance, which is not necessarily true for many image retrieval applications. One attempt to solve the indexing problems is to use hierarchical indexing scheme based on the Self-Organization Map (SOM) proposed in [21].

\section{CONCLUSION}

This paper investigated various feature extraction algorithms in CBIR. A study of different color and texture features for image retrieval in CBIR is performed. Numerous methods are available for feature extraction in CBIR. They are identified and studied to understand the image retrieval process in the CBIR systems. Studies made on experiment results show that the method based on hybrid combination of color and texture features has higher retrieval accuracy than the other methods based on single feature extraction. Color moments, color histograms, color correlogram and gabor texture are considered for retrieval. It is difficult to claim that one feature is superior to others. The performance depends on the color distribution of images. The combination of color descriptors produces better retrieval rate compared to individual color descriptors. Color moments and color histogram features can be combined to get better results. Color histograms and correlograms can be combined retaining advantages of histograms with spatial layout. Similarly, Texture feature can be combined with color moments or color histogram to get accurate results for image retrieval. From the studies, it is found that only one color feature or texture feature is 
not sufficient to describe an image. There is considerable increase in retrieval efficiency when both color and texture features are combined.

\section{REFERENCES}

[1] Kenneth R. Castleman, (1996)“Digital Image Processing” . Prentice Hall .

[2] A. Blaser, (1979) "Database Techniques for Pictorial Applications", Lecture Notes in Computer Science, Vol.81, Springer Verlag GmbH.

[3] Ryszard S. Chora's(2007) "Image Feature Extraction Techniques and their Applications for CBIR and Biometrics Systems“" International journal of biology and biomedical engineering Issue 1, Vol. 1.

[4] M. Stricker, and M. Orengo, (1995) "Similarity of color images," SPIE Storage and Retrieval for Image and Video Databases III, vol. 2185, pp.381-392.

[5] J. Huang, et al., (1997) "Image indexing using color correlogram," IEEE Int. Conf. on Computer Visionand Pattern Recognition, pp. 762-768, Puerto Rico.

[6] Deepak S. Shete1, Dr. M.S. Chavan (2012) "Content Based Image Retrieval: Review” International Journal of Emerging Technology and Advanced Engineering ISSN, Volume 2, pp2250-2459.

[7] Fazal Malik , Baharum Baharudin(2013) "Analysis of distance metrics in content-based image retrieval using statistical quantized histogram texture features in the DCT domain”, Journal of King Saud University -Computer and Information Sciences Vol 25 ,pp.207 -218.

[8] Manimala Singha and K.Hema chandran(2012) "Content based image retrieval using color and texture "Signal \& Image Processing : An International Journal (SIPIJ) Vol.3, No.1, pp.39-57.

[9] Md. Iqbal Hasan Sarker and Md. Shahed Iqbal (2013) "Content-based Image Retrieval Using Haar Wavelet Transform and Color Moment” Smart Computing Review, vol. 3, no. 3, pp.155-165.

[10] MS. R. Janani, Sebhakumar.P (2014) "An Improved CBIR Method Using Color and Texture Properties with Relevance Feedback International Journal of Innovative Research in Computer and Communication Engineering Vol.2, Special Issue 1.

[11] K. Arthi, Mr. J. Vijayaraghavan (2013) "Content Based Image Retrieval Algorithm Using Colour Models" International Journal of Advanced Research in Computer and Communication Engineering Vol. 2, Issue 3.

[12] Shengjiu Wang (2001) “A Robust CBIR Approach Using Local Color Histograms” Technical Report TR 01-13.

[13] J. Zhang, G. Li, S. He, "Texture-Based Image Retrieval by Edge Detection Matching GLCM", The 10th IEEE International Conference on High Performance Computing and Communications.

[14] A. K. Jain, and F. Farroknia, (1991) "Unsupervised texture segmentation using Gabor filters," Pattern Recognition, Vo.24, No.12, pp. 1167-1186.

[15] YogitaMistry, Dr.D.T. Ingole (2013) " Survey on Content Based Image Retrieval Systems" International Journal of Innovative Research in Computer and Communication Engineering Vol. 1, Issue 8.

[16] S. Mangijao Singh , K. Hemachandran (2012) “Content-Based Image Retrieval using Color Moment and Gabor Texture Feature" IJCSI International Journal of Computer Science Issues, Vol. 9, Issue 5, No 1, pp.299-309.

[17] Julie M. David, Kannan Balakrishnan, (2014), "Learning Disability Prediction Tool using ANN and ANFIS” , Int. J.of Soft Computing, Springer Verlag Berlin Heidelberg, ISSN 1432-7643 (online), ISSN 1433-7479 (print), DOI: 10.1007/s00500-013-1129-0, 18 (6), pp 1093-1112.

[18] M. Flickner, H. Sawhney, W. Niblack, J. Ashley, Q. Huang, B.Dom, M. Gorkani, J. Hafner, D. Lee, D. Petkovic, D. Steele, and P. Yanker, (1995) "Query by image and video content: The QBIC system." IEEE Computer, Vol.28, No.9, pp. 23-32.

[19] N. Beckmann, et al, (1990) "The R-tree: An efficient robust access method for points and rectangles," ACM SIGMOD Int. Conf. on Management of Data, Atlantic City.

[20] J. Vendrig, M. Worring, and A. W. M. Smeulders, (1999) "Filter image browsing: exploiting interaction in retrieval," Proc. Viusl'99: Information and Information System.

[21] H. J. Zhang, and D. Zhong, (1995) "A Scheme for visual feature-based image indexing," Proc. of SPIE conf. on Storage and Retrieval for Image and Video Databases III, pp. 36-46, San Jose. 


\section{AUTHORS}

Shereena V.B.received her MCA degree from Bharathidasan University, Trichy, India in 2000. During 2000-2004, she was with Mahatma Gandhi University, Kottayam, India as Lecturer in the Department of Computer Applications. Currently she is working as Asst. Professor in the Department of Computer Applications with MES College, Aluva, Cochin, India. Her research interests include Data Mining and Image Processing.

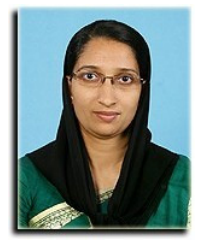

Dr. Julie M. Davidcompleted her Masters Degree in Computer Applications and Masters of Philosophy in Computer Science in the years 2000 and 2009 in Bharathiyar University, Coimbatore, India and in Vinayaka Missions University, Salem, India respectively. She has also completed her Doctorate in the research area of Artificial Intelligence from Cochin University of Science and Technology, Cochin, India in 2013. During 2000-2007, she was with Mahatma Gandhi University, Kottayam, India, as Lecturer in the Department of Computer Applications. Currently she is working as an Assistant Professor

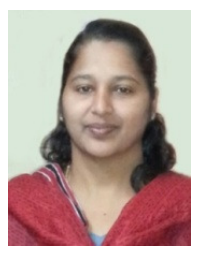
in the Department of Computer Applications with MES College, Aluva, Cochin, India. She has published several papers in International Journals and International and National Conference Proceedings. Her research interests include Artificial Intelligence, Data Mining, and Machine Learning. She is a life member of International Association of Engineers, IAENG Societies of Artificial Intelligence \& Data Mining, Computer Society of India, etc. and a Reviewer of Elsevier International Journal of Knowledge Based Systems. Also, she is an Editorial Board Member of various other International Journals. She has coordinated various International and National Conferences. 